\title{
TURISMO EN LIMÓN: DATOS Y ELEMENTOS DE ANÁLISIS SOBRE LA DINÁMICA DE LAS ACTIVIDADES LABORALES. EL CASO DEL BARRIO PACUARE UBICADO EN EL CANTÓN CENTRAL
}

\author{
TOURISM IN LIMON: DATA AND ELEMENTS OF ANALYSIS ABOUT \\ THE DYNAMIC OF THE LABOR ACTIVITIES. THE CASE OF BARRIO \\ PACUARE, LOCATED IN THE CENTRAL CANTON
}

\section{Pablo Carballo Chaves*}

RESUMEN

El artículo pretende como principal punto mostrar los resultados de investigación que se desarrollaron en el marco del Proyecto Centros Históricos del Turismo, realizado por la Universidad de Costa Rica (UCR) ${ }^{1}$ que viene funcionando desde hace más de dos años. Después de una depuración técnica/teórica de la base de datos surgida en un censo, en el Barrio Pacuare del cantón central de la provincia Limón en Costa Rica, se desarrolló una tipología laboral que condensa los siguientes segmentos: Turismo, Público, Formal, Informal-Doméstico y Otros, para el caso de la base de individuos. En el caso de la base de datos de hogares, se le agrega la categoría de No ocupados. Se busca presentar datos para la discusión y la reflexión sobre el turismo, desde una tipología laboral que establezca una capacidad relacional del turismo con respecto a lo que acontece laboralmente en el Barrio Pacuare y en Limón, en la medida de lo posible.

PALABRAS CLAVE: LIMÓN, COSTA RICA * TURISMO * MERCADO DE TRABAJO * COMUNIDADES * OPINIÓN

\section{ABSTRACT}

This paper brings the main results achieved by the proyect: Centros Históricos del Turismo (UCR) Tourism historial centers (UCR) operating for more than two years. After a technical cleaning, obtained several data from the Pacuare neighborhood Barrio Pacuare located in the Limon central canton in Costa Rica. It has been created a laboral typology with these

Universidad de Costa Rica (UCR), Universidad Estatal a Distancia (UNED) y Facultad Latinoamericana de Ciencias Sociales (FLACSO), Costa Rica. carballopablo@gmail.com MBA. Ivonne Lepe Jorquera, Lic. Cynthia Arrieta Brenes y al Bach. Bladimiro Argueta (todos, funcionarios de la Sede de Limón/UCR). 
segments: Tourism, Public, Formal, Informal-Domestic, and Others, all this segments are for the individual data base. In the case of the home data base it has been incorporated: unoccupied. We present data that help to the discussion and reflection about tourism, and labor situation of Limón, especially in the Pacuare case.

KEYWORDS: LIMON, COSTA RICA * TOURISM * LABOR IN MARKET * COMMUNITIES * OPINION

\section{INTRODUCCIÓN}

En la Universidad de Costa Rica se viene realizando desde febrero de 2011, un sistemático estudio sobre el turismo en dos zonas sociogeográficas costarricenses, que tienen tanto similitudes como diferencias. El proyecto que enmarca la construcción de los datos que se desarrollarán en el presente artículo se denomina: Centros Históricos del Turismo: Los casos de Puntarenas y Limón ${ }^{2}$. Para ello se contó con el trabajo colectivo de la Escuela de Sociología de la Sede Rodrigo Facio, la Sede de Puntarenas $y$ la Sede de Limón, todas parte de la Universidad de Costa Rica, y bajo un sistema denominado intersedes, el cual busca ahondar en la investigación y la conexión tanto inter-universitaria, como la relación entre las distintas partes del país. Si bien, el proyecto involucra a los dos puertos de Costa Rica, en este artículo solamente se profundizó en el contexto de Limón, particularmente en el distrito central de dicha provincia y puntualmente, en el Barrio Pacuare. Los datos que se proporcionan surgen de la recopilación del material en dicho barrio, por lo que se propone una profundización en contexto barrio/comunal, pero que busque, al menos de manera introductoria, dar ciertas líneas generalizadoras. Esto sin omitir, con conocimiento de fondo, las particularidades geográficas $y$ socio-comerciales de otras zonas de la provincia de Limón, como sus zonas de playa o sus sistemas y canales acuíferos internos. Sin embargo, es en el Barrio Pacuare donde se concentra la atención del documento, además del interés principal que es presentar la información/datos $y$ de manera tangencial, asimilar algunas breves explicaciones.

2 Proyecto adscrito a la Vicerrectoría de Investigación de la Universidad de Costa Rica. Número de inscripción: 211-B0-246.

\section{BREVE CONTEXTUALIZACIÓN DEL TURISMO EN LA ZONA DE LIMÓN}

El turismo es una de las actividades que se ha desarrollado con más fuerza en distintas partes del planeta. Como parte de una dinámica de expansión, tanto de inversión de capitales, como de dinámica de movilización de personas, el turismo se explica dentro de la reconstrucción de las formas productivas, donde América Latina fue una de las zonas donde se concentró un desarrollo de esta actividad económica. Asimismo, en Centroamérica, la actividad tuvo un importante impulso durante los años 90 , en Costa Rica, por eso:

... hay que tomar en cuenta el turismo. Su dinamismo se refleja tanto en el hecho que en 1990 ingresaron en la región millón y medio de turistas, flujo que se incrementó en casi un millón más, siete años más tarde. Destacan dos países, Guatemala y Costa Rica. Pero si el primero de ellos, a inicios de la década, recibía un poco más de medio millón de visitantes, superando levemente al segundo, ya para 1997, Costa Rica había alcanzado los 800000 turistas, cifra superior a la guatemalteca (similar a la de inicio de la década) (canatur, 1998, citado en Pérez, Andrade-Eekhoff, Bastos y Herradora, 2004: 48).

En Costa Rica, esta actividad se ha estado desarrollando desde hace tiempo $y$ donde uno de sus más importantes momentos de desarrollo fue durante los años 80, pero su momento de desarrollo masivo y global fue durante los años 90, ya que crece la actividad, pero esta vez a un nivel amplio de inversión extranjera. Sin embargo, uno de los más importantes puntos de 
desarrollo estuvo asociado a lo que se denomina turismo interno ${ }^{3}$. De ahí que una de las justificaciones del proyecto fue rescatar estos clásicos destinos turísticos nacionales, valorar sus circunstancias (que se explicitarán más adelante) $y$ generar una serie de datos que colaboren con la comprensión del fenómeno del turismo en su condición económica y socio-cultural.

Señalado lo anterior, es fundamental comprender cómo la lógica de encadenamiento del comercio y la productividad mundial, también incorporó al turismo dentro de esta dimensión de la capitalización del ocio en distintas facetas y en la cual aprovechó los distintos escenarios y servicios para construir toda una lógica de desarrollo comercial. La provincia de Limón se presenta como un ejemplo de esta penetración del capital internacional y la adecuación de sus playas, así como, sitios naturales y sociales, para albergar, además del mencionado turismo local, la entrada de extranjeros en diferentes niveles, cantidades y actitudes.

La provincia, a partir del turismo - entre otras actividades - ha tenido que reestructurar vivencias a nivel de sistemas y de administración de recursos que no solamente podrían traer ventajas, sino que han traído también desventajas, como por ejemplo, con los recursos hídricos; recursos que también pueden ser una fuente importante bajo lo que se ha denominado el "ecoturismo" (Bonilla, Pérez y Michelena, 2005). Además, lo concerniente a la infraestructura en distintas zonas, ha sido una de las circunstancias que más ha sido abordada, tanto por su desarrollo y su inversión como por sus problemáticas a nivel ambiental (Murillo, 2006).

Se pueden encontrar diferentes puntos para analizar el espectro del turismo en la zona; sin embargo, se puede puntualizar en el aspecto jurídico/ambiental (García y Lepe, 2009 y Ruiz, 2006) y de aprovechamiento económico-comercial (Román, Jiménez y Molina, 2004), como dos de las facetas que han tenido atención en los

3 Personas del país visitando zonas del mismo país y creando tanto rutinas de sitios predilectos, como rotando los diferentes destinos que se fueron presentando $y$ desarrollando como zonas turísticas. Ejemplo de esto fueron los puertos de Limón y Puntarenas, así como, la provincia de Guanacaste. últimos años y forman parte de un papel crítico de dicha actividad en la provincia, así como a nivel nacional e internacional. Por otro lado, es fundamental conocer que dentro de las dificultades de surgimiento de la región, se encuentra el ámbito de la desigualdad socio-cultural, históricamente presente en donde:

... la atracción de Limón como destino turístico no ha estado exenta de contradicciones prejuicios muy fuertes $y$ arraigados entre la población blanca y mestiza del resto del país, especialmente la del Valle Central. Antes de 1949 a los habitantes negros de la provincia se les prohibía el paso hacia el centro del país, impidiéndoles viajar después de Turrialba, en dirección hacia San José. Simbólicamente debe destacarse que fue hasta 1964 que se agregó una estrella al escudo nacional, en representación justamente de Limón; la sétima provincia (Cordero, 2011: 32).

Así se mantiene un perfil crítico de las dificultades que presenta la región en un sentido más completo que solamente el turístico, aunque sea este el punto de atención del artículo.

\section{RESUMEN CRÍTICO DEL PROCESO METODOLÓGICO}

La información estadística que se presenta en el desarrollo del artículo surge del censo realizado en el Barrio Pacuare, en el año 2011. Se seleccionó dicho lugar por tres razones: 1 ) ser parte del cantón central de la Provincia; 2) bajo la consideración de ser parte de un sitio de turismo histórico y 3) principalmente, porque después de hacer un sondeo inicial en empresas $y$ sitios, se dio con que es Pacuare, el lugar que alberga el mayor porcentaje de gente relacionada con alguna actividad turística. En el censo participaron diferentes miembros de la Universidad de Costa Rica, entre ellos: estudiantes, docentes y los respectivos investigadores, en diferentes calidades: constructores de la encuesta, así como, quienes la fueron a aplicar. Es así como una primera y segunda etapa, se pueden condensar en tres actividades fundamentales: 
selección del lugar, construcción del instrumento de recolección de información y la respectiva aplicación.

La tercera etapa fue el análisis de la información, la cual se realizó en los primeros meses del año 2012. Para ello, previamente se realizó el ingreso de la información a las bases de datos en el programa denominado spss (Statistical Package for Social Sciences). Desde ahí se empezó un trabajo de lectura introductoria a los datos, que posteriormente se convirtió en un trabajo de limpieza de las bases, además de conocimiento general de las posibilidades de análisis que existía.

Una de las indicaciones fundamentales para tener presente es que en el instrumento de encuesta aplicado se albergaba la necesidad de rescatar material tanto individual (por persona), como a nivel de hogares. De esta forma, el proceso de depuración de la base se hizo para las dos bases de datos, cada una con material relativo a lo individual (con un nro. de 276) y de hogares (con un nro. de 281$)^{4}$. Pero sin embargo, tienen mucha relación, ya que en ciertas situaciones se necesitó incorporar elementos de la base individual en la de hogares, para poder reconstruir categorías, las cuales al final resultaron en la construcción de la tipología de actividades laborales que es uno de los más importante resultados del proyecto, junto con los datos y las interpretaciones surgidas.

Después de depurar, revisar y solucionar problemas diversos ${ }^{5}$ con los datos, se llegó a la construcción de la tipología, tanto para los

$4 \quad$ Los números de individuo y hogares son distintos debido a los procesos de "limpieza" y depuración de las bases de datos, así como a la selección de los casos donde se albergaban criterios relativos al aporte turístico en el barrio seleccionado. El censo consistió en un total de: 1200 personas consultadas, las cuales se procesaron en aras de depurar la base de datos y con el fin de construir la tipología, así como, la construcción de los cuadros para el análisis.

$5 \quad$ Entre otros problemas a mencionar, se encuentra el tema de problemas de encuestas con espacios vacíos (que redundaban en resolver si fue: falta de datos, error del encuestador o error del digitador). Además, recordar analizar si la información trabajada era coincidente y con lógica en la reflexión teórica. casos de Limón como de Puntarenas. La tipología de actividades laborales en el caso de Limón se puntualizó en las siguientes funciones: A. Turismo, B. Sector Público, C. Sector Formal y D. Sector Informal Doméstico; a los cuales se les adjunta otras dos categorías: E. Otros ${ }^{6} y$ F. No ocupados ${ }^{7}$. Estos dos últimos para complementar los datos y las cantidades dentro del proceso de clasificación.

La construcción de esta tipología redunda en dos situaciones: 1) Hace posible el manejo de datos (claramente con la inclinación hacia las actividades laborales, pero no exclusivamente, como se verá más adelante) y 2) Permite compartir una posición teórico/metodológica, la cual podría permitir continuar el proyecto $y$ sus indagaciones, como también, ser incorporada o repensada en otros contextos: locales, nacionales o regionales.

\section{ALGUNAS CONSIDERACIONES TEÓRICAS}

La investigación tiene muchas salidas analíticas; sin embargo, se concentrará en reforzar la reflexión sobre el tema de las actividades laborales en el Barrio Pacuare. No obstante, para lograr esta centralización crítica, se fortalecerá el análisis teniendo algunas precisiones sobre el concepto clave que es el "turismo", así como algunas notas sobre la idea de "comunidad", como conceptos que orientan una discusión crítica sobre la complejidad del entorno limonense estudiado.

\section{A) TURISMO: LIMÓN GLOBALIZADO}

Diferentes enfoques pueden reconstruir una reflexión sobre el turismo; sin embargo, se optó por reconocer a esta dinámica como una faceta económica importante que se desarrolla con suma fuerza dentro de la lógica capitalista de acumulación actual.

6 Otros: esta categoría involucra trabajos o actividades que no se pudieron incluir como nueva categoría o dentro de las cuatro categorías básicas antes señaladas. En el caso de Puntarenas, se agrega una categoría más a las básicas, la Pesca. Sin embargo, en el caso de Limón, esta categoría no surgió.

7 La categoría "No ocupados" funciona exclusivamente para la tipología en el caso de los hogares. 
De modo que se puede suponer que la historia del turismo en el seno de la sociedad capitalista, empieza como una actividad que es posible, dado un mayor desarrollo de los servicios en particular del transporte $y$ de los alojamientos, pero presumiblemente, en lo que tiene que ver con la naturaleza social del turismo, la posibilidad de su disfrute estaba principalmente limitada a las clases altas de la sociedad (Cordero, 2004: 18).

La globalización, las necesidades de acumular y la participación social extendida en dichas tareas de acumulación, expandieron la posibilidad del turismo en varios sentidos: un sentido de aprovechamiento a partir del ocio, además de un sentido empresarial, desarrollando compañías y corporaciones; $y$ de reapropiarse productivamente de los escenarios y recursos naturales que se tenían a disposición, como ha sucedido en muchos de los casos del turismo centroamericano y costarricense.

En el contexto mundial, el turismo también se ha visto permeado por los reacomodos productivos y del manejo político y económico. En esa línea, la globalización obliga a conectar las dinámicas internas del aprovechamiento $y$ explotación de recursos con los mercados internacionales. Sin embargo, está dinámica también reorienta la organización de los trabajadores en el plano del turismo, también obliga a adaptarse, tanto a las demandas de mercado, como a diversos requerimientos comerciales.

Como el cambio hacia la producción flexible se ha dado en un contexto de globalización de la producción, lo que ha conllevado a que muchas firmas dispersen la producción y la distribución a una escala global (...) no estaría claro si la política de la especialización flexible se ve acompañada de una valorización de las remuneraciones obreras o más bien, lo que es más probable, estaría asociada con los procesos de flexibilización laboral que se caracterizan, principalmente en los países pobres por una profundización de la precarización de las formas de contratación, las condiciones de trabajo $y$ de los salarios (Cordero, 2004: 42).
Si bien es cierto, el turismo tiene una importante conexión con la globalización y la acumulación, también nuevas formas turísticas han mostrado una diversa manera de aprovechar $y$ reconstruir las formas organizativas de las comunidades. El "ecoturismo" 8 el "etnoturismo", entre otras posibles, muestran esa diversificación cultural y social, así como, las posibilidades de desarrollo con perspectivas distintas a la gran explotación mundial. Muchas de estas lógicas de aprovechamiento turístico se encuentran iniciadas $y$ desarrolladas en la provincia de Limón, lo que muestra lo complejo del escenario, ya que unas dinámicas pueden cohabitar en algún sentido, pero otras pueden ser inclusive contradictorias con un modelo imperante.

Es así como el turismo, de manera breve y básica, es una dinámica que tiene un entronque fundamental en el espacio socio-laboral, donde lo natural, lo cultural y lo social en general, entran como recurso de explotación o recurso de apoyo, para brindar experiencias de ocio en diferentes medidas y contextos. Donde la organización de la vida en las comunidades en muchas oportunidades, se recrea para satisfacer demandas de ocio externas y que termina por moldear y acondicionar la propia población, debido a las demandas de turistas y de inversión que intervienen también en toda la reconstrucción socio-estructural de las regiones. Además, donde la actividad turística viene a participar cada vez con más fuerza de la estructuración que existe en la sociedad limonense, permeada por la lógica desarrollista del Estado costarricense que se dio durante los años 70 y 80, donde el apogeo formalista captó importantes grupos de personas, pero que en la actualidad, ha encontrado un estancamiento riesgoso y preocupante. Esto obligó a las personas, junto con las que nunca ingresaron en las

\footnotetext{
8 Dinámica turística que se asocia con los procesos ecológicos, así como, respeto y organización hacia la naturaleza, su explotación y su importancia global (cfr. Vanegas, 2006).

9 Dinámica turística que tiene su particularidad en la exposición de las diferencias culturales/étnicas, particularmente, las indígenas, en las comunidades que habitan ciertas zonas geográficas (cfr. Pilquiman y Skewes, 2010).
} 
filas del Estado, a buscar trabajos en lo privado $y$ en muchos de los casos $-y$ últimamente con más fuerza- en la informalidad.

\section{B) LA COMUNIDAD: ESPACIO CULTURAL}

La tradición de la idea conceptual de comunidad es ya de larga data en sociología y dentro de dicha reflexión clásica, no está exenta de problemáticas analíticas. Desde la diferencia que establecía Ferdinand Tönnies entre sociedad $y$ comunidad $^{10}$, el tema de la comunidad ha estado asociado a los elementos de lazos de familiaridad, cercanía, amistad e históricos. Además, el criterio de comunidad puede estar referido, tanto a linderos culturales como nacionalistas (cfr. Poviña, 1949), entre otros. Sin entrar en esta discusión, se puede rescatar, para orientar la visión de integración en el sitio estudiado, que la reflexión de las actividades laborales con énfasis en el turismo está referida a una zona/barrio que se ancla en una lógica que se podría señalar como de comunidad, siguiendo los elementos arriba señalados de Tönnies ${ }^{11}$. Sin embargo, los nuevos patrones globales de mercado y sociedad hacen replantear el papel de las comunidades en dicho entramado mundial, máxime que el turismo dentro de la dimensión capitalista de acumulación se ha posicionado con fuerza en sitios que no tienen necesariamente la tradición de globalidad que otros. En este sentido:

En Giddens, la comunidad estará relacionada al problema del registro reflexivo y la reflexividad institucional en aumento tanto en la temprana modernidad, como aún más en condiciones de modernidad tardía. Las comunidades que emergen o re-emergen solo podrán ser comprendidas si se establecen las correlaciones necesarias con los conceptos de saber mutuo y agencia (Bialakowsky, 2010: 24).

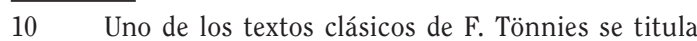
precisamente: Comunidad y sociedad (en alemán: Gemeinschaft und Gesellschaft), en 1887.

$11 \quad$ El análisis de F. Tönnies es mucho más profundo $y$ denso, pero se omite para posicionar el artículo en un lindero más matizado y con una exposición sintética de los datos.
Es clarificador establecer un criterio sobre la comunidad, ya que al estudiar un barrio de una zona en particular, se pueden encontrar patrones o circunstancias relativas a lazos específicos de convivencia humana - como lo podría ser a nivel básico: sobrevivir ${ }^{12}$ - $y$ que además podrían explicar ciertas lógicas de comportamiento $y$ de acomodos en términos de la apropiación de una dinámica laboral, como lo sería en este caso, el turismo en Limón. Por esta razón, el tema de los saberes mutuos, aunque no se desarrolla en este artículo, sería útil si se concentra en la faceta de apropiaciones prácticas por parte de las personas que viven en ciertas comunidades con potenciales desarrollos turísticos. Sin embargo, esto no garantiza el uso eficiente, ni el aprovechamiento que redunde en la misma comunidad. En el caso de la "agencia", se estaría relevando el caso de conciencia (individual y colectiva) respecto a cómo hacer para construir, defender y revalorar las relaciones de productividad, consumo y servicios, que del turismo se pueden generar. Aunque tampoco se desarrolla el concepto de agencia, sí es relevante mantener la idea de un posible conocimiento y gestión propia, no abstraída de las conexiones políticas, ni las socio-económicas, pero sí performativas de propias decisiones y prácticas que definan perspectivas propias con resultados mucho más alentadores.

La comunidad es entonces, en lo que concierne en este momento, una relación que preserva rasgos de convivencia histórica $y$ vivencial, con una zona o espacio socio-geográfico particular y que entra en diálogo directo con las personas que lo habitan, moldeando

$12 \quad$ Clarificar también, si bien algunas corrientes sociológicas unen la idea de comunidad con las corrientes organicistas o el contractualismo. En este documento no se llevó por ese camino ( $y$ de hacerlo, se unificaría más con una versión contractualista, pero amortiguada por ciertas aclaraciones) y se deja el concepto de comunidad, como una forma organizativa cultural que tiene lógicas internas, así como, convivencias con el exterior, y que se orienta a la construcción de patrones de vivencia económica creativa, que no deja de lado las relaciones jerárquicas y en conexión con su vivencia geográfica. 
uno al otro, además, con un nivel de "agencia" (relativo) con la cual poder reconstruir la vivencia y la apropiación de las relaciones, recursos y perspectivas sociales. Sin embargo, complementado con la modernidad y los impulsos capitalistas, la comunidad es un espacio de confrontación y debate donde viejos y nuevos paradigmas socio-económicos se desarrollan; así como, grupos distintos dentro y fuera de la comunidad establecen criterios para la apropiación y uso de lo que en la comunidad (socio-geográficamente y recursos en general) se puede aprovechar, en la mayoría de los casos desde la mirada económico-comercial.

\section{EXPOSICIÓN DE ELEMENTOS INDIVIDUALES DEL TURISMO EN LIMÓN}

Como se rescató en el acápite metodológico, en el censo se logró recoger información individual sobre la población estudiada del Barrio Pacuare. En dicha sección, según los datos individuales de las personas, se recogió información como edad, escolaridad, entre otros. En el cuadro 1, se presentan las cantidades de personas según las categorías de la tipología; mientras que en el cuadro 2, se presenta información más detallada sobre las variables clave respecto a los individuos $y$ a las respectivas categorías, tanto en promedios como en porcentajes.

CUADRO 1

TIPOLOGÍA LABORAL DE LA BASE DE INDIVIDUOS EN EL BARRIO PACUARE SEGÚN SU NÚMERO DE ENTREVISTADOS EN ÁREA DE ACTIVIDAD 2011-2012

\begin{tabular}{cccccc}
\hline TURISMO & PÚBLICO & FORMAL & INFORMAL-DOMÉSTICO & OTROS & TOTAL \\
\hline N: 34 & N: 76 & N: 95 & N: 52 & N: 19 & N: 276 \\
\hline
\end{tabular}

Fuente: Elaboración propia en el marco del proyecto Centros Históricos del Turismo. Censo 2011.

En el cuadro 1, se muestra una importante presencia de las áreas de ocupación en el sector público y formal, según la recolección de información, siendo el sector formal el ámbito donde más personas se lograron detectar y refle- jando de entrada, la importancia del sector en la dinámica de trabajo del barrio. En el cuadro 2, se presenta la distribución de datos según características de las personas, especificando también en el carácter de sus particularidades laborales. 


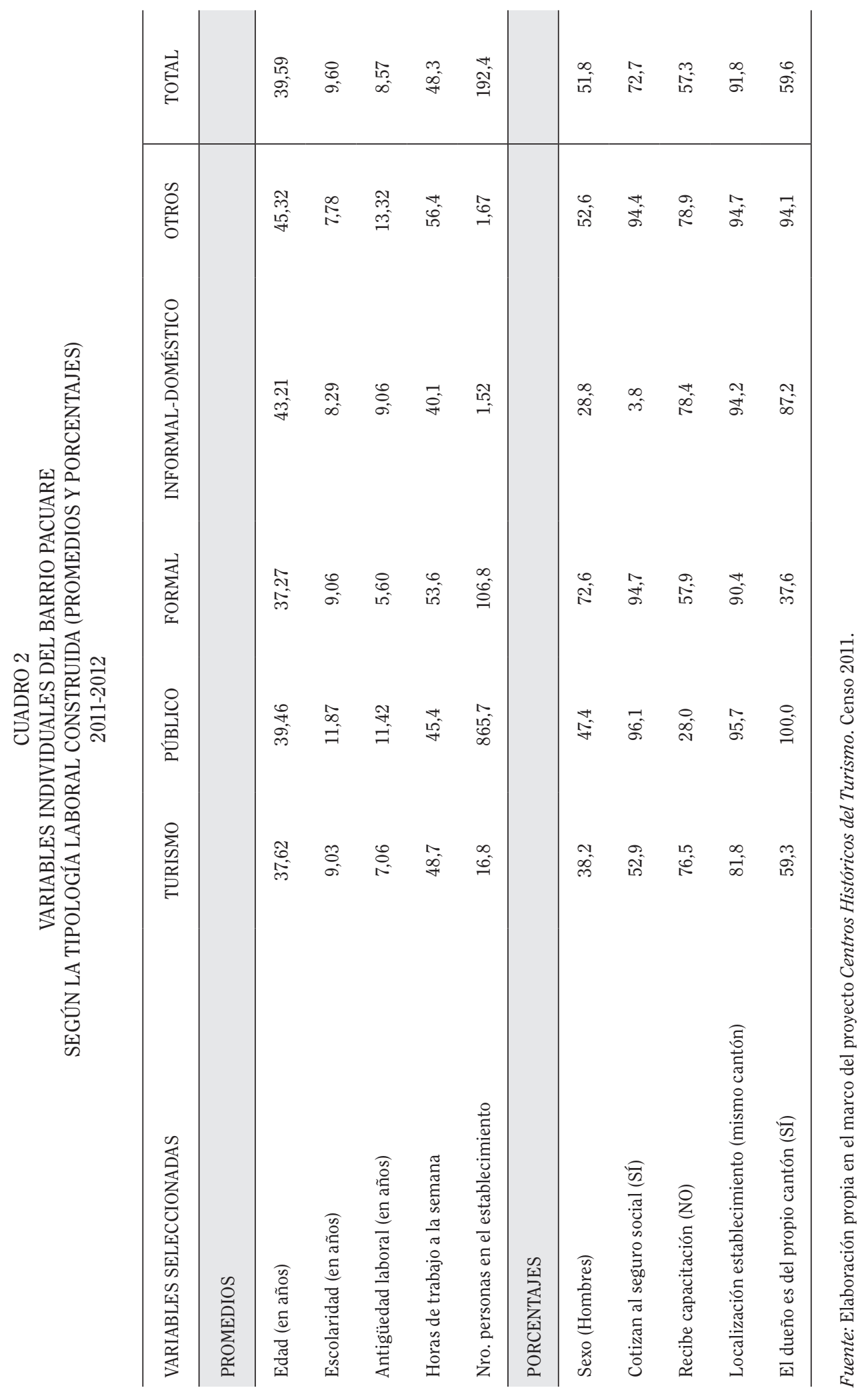


En el cuadro 2 se presenta una distribución de edades donde el peso más alto de edad se ubica en la categoría "Informal-Doméstico" (43 años promedio). Se debe recordar que al realizar el cruce con la variable sexo, el grueso de las personas que se desempeñan en dichos trabajos son mujeres (71\%) y además, presentando también uno de los segmentos con personas de menor escolaridad (8,3 años promedio). Por el contrario, el sector "Formal" alberga, en cuestión de sexo, una mayoría de hombres (72 \%) y con la edad más baja de toda la tipología en años promedio (37 años). Sin embargo, la escolaridad no resulta tan alta como lo es en el sector "Público", que por ciertas lógicas técnicas, busca un perfil escolar más alto. En el caso de la escolaridad, el sector "Formal" se equipara con el caso del "Turismo", donde en ambos casos los años de escolaridad es de 9 años. Además, en "Turismo" se presenta también una de las edades promedio más jóvenes (37 años), que también se equipara con la del sector "Formal".

Después de esta panorámica inicial de contrastes significativos, en el caso del "Turismo" se puede observar que representa una de las actividades que más tiempo, en horas de trabajo, emplea a la semana (49 horas promedio), solo superado por el sector "Formal" (53 horas promedio). Se aprecia que en el caso del "Turismo" y del sector "Informal-Doméstico" son de los que menos porcentaje de capacitación tienen (Turismo/ No: $76 \%$ y Informal-Doméstico/No: 78\%); lo que se puede orientar a la comprensión de una alta informalidad y dinámicas pro- pias de la comunidad o de las personas que dependen de dichos nichos de trabajo. La cotización al seguro social de las personas del sector "Turismo" es del 52\%. Esto podría tener algún criterio comparativo para desarrollar, si se apela también que en este sector la mayoría son mujeres (61\%) y que posiblemente tienen cargas familiares, como se verá más adelante en los cuadros referentes a las comparaciones en los hogares.

En el cuadro 4 de la siguiente sección, se observa que la mayoría de las jefaturas familiares son mujeres y para el caso del "Turismo" es de $62 \%$, en donde las mujeres son las jefas de hogar, según el resultado de los datos de los instrumentos aplicados. En esta línea de jefaturas de mujeres, el "Turismo" es superado por el sector "Informal-Doméstico" (70\%). En el caso de los sectores "Público" y "Formal" se invierte, ya que son los hombres los que tienen más significación porcentual en las jefaturas de los hogares, destacando el caso del sector "Formal", al ser el más alto en presencia masculina (75\%).

\section{EXPOSICIÓN DE DATOS SOBRE HOGARES Y TURISMO EN LIMÓN}

Así como la base de individuos tienen sus propias cantidades, la base de hogares presenta su distribución en el cuadro 3. En esta tenemos un total de 281 hogares $y$ donde el peso del "Turismo" es el más bajo respecto a las restantes categorías. El caso del sector "Formal" sigue siendo el ámbito laboral donde más personas se registraron.

CUADRO 3

TIPOLOGÍA LABORAL DE LA BASE DE HOGARES EN EL BARRIO PACUARE

POR NÚMERO DE ENTREVISTADOS

SEGÚN ÁREA DE ACTIVIDAD

2012

\begin{tabular}{ccccccc}
\hline TURISMO & PÚBLICO & FORMAL & $\begin{array}{l}\text { INFORMAL- } \\
\text { DOMÉSTICO }\end{array}$ & OTROS & NO OCUPADO & TOTAL \\
\hline $\mathrm{N}: 29$ & $\mathrm{~N}: 54$ & $\mathrm{~N}: 64$ & $\mathrm{~N}: 38$ & $\mathrm{~N}: 42$ & $\mathrm{~N}: 54$ & $\mathrm{~N}: 281$ \\
\hline
\end{tabular}

Fuente: Elaboración propia en el marco del proyecto Centros Históricos del Turismo. Censo 2011. 
Como se reflejó en líneas anteriores, la temática de las jefaturas femeninas en el caso del "Turismo" es importante recalcarla, ya que junto con el segmento de "InformalDoméstico", son las que lideran las jefaturas de los hogares, esto según el cuadro 4. Si se ahonda en este aspecto comparativo entre estos dos segmentos de la tipología, se observa que también son los que presentan la escolaridad más baja, 9 años promedio para "Turismo" y 7,7 años promedio para "Informal-Doméstico", esto sin contar la categoría "No ocupados". El mayor contraste educativo (escolaridad), de nuevo, se encuentra en el segmento "Público" con un promedio en años de 11.

Al momento de analizar la condición de actividad del o de la jefa de hogar, se encontró que en el caso del "Turismo" la condición de mantenerse ocupado es de $86 \%$, parecida a los restantes segmentos, "Público" y "Formal".
Pero en caso del segmento "Informal-Doméstico", crece la condición de actividad hasta el $92 \%$. También "Turismo" y el segmento "Informal-Doméstico" coinciden en ser las categorías con los menores ingresos reportados por mes en el hogar ("Turismo": \$247 541 y "InformalDoméstico": \$171 182). Este tipo de comparaciones orientan para comprender la relación, o al menos, las características de estos dos segmentos ("Turismo/ Informal-Doméstico") en los cuales se notan los índices más bajos en temáticas de formación y de asistencia estatal o social.

Por otro lado, otro punto que se pretende rescatar es que en el instrumento se encuentra información relativa a las consideraciones/opiniones de las personas del Barrio sobre lo que el turismo representa y en lo que el turismo podría estar relacionado con situaciones sociales de la comunidad. El cuadro 5 y el cuadro 6 muestran en datos algunas de esas percepciones. 


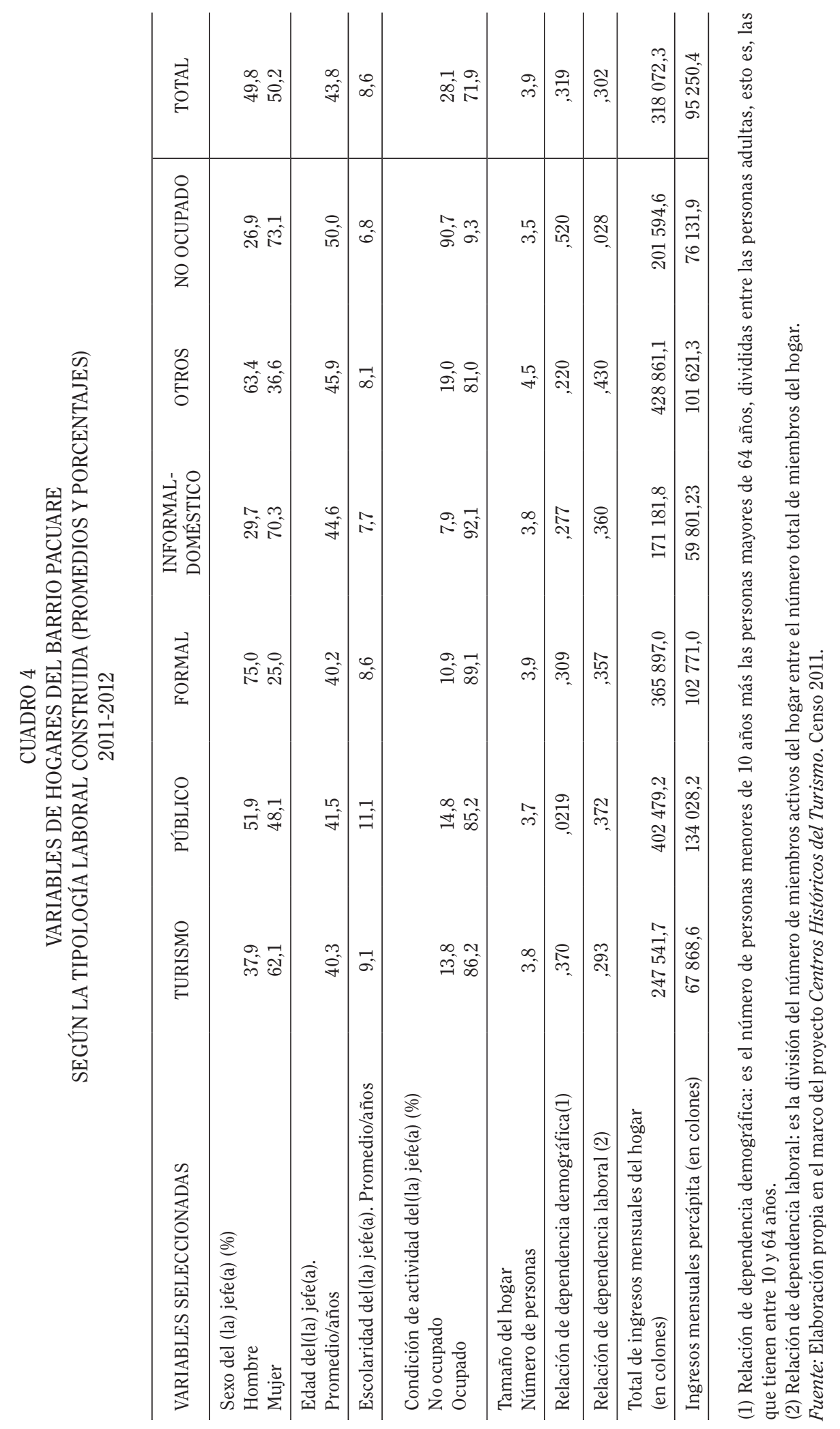




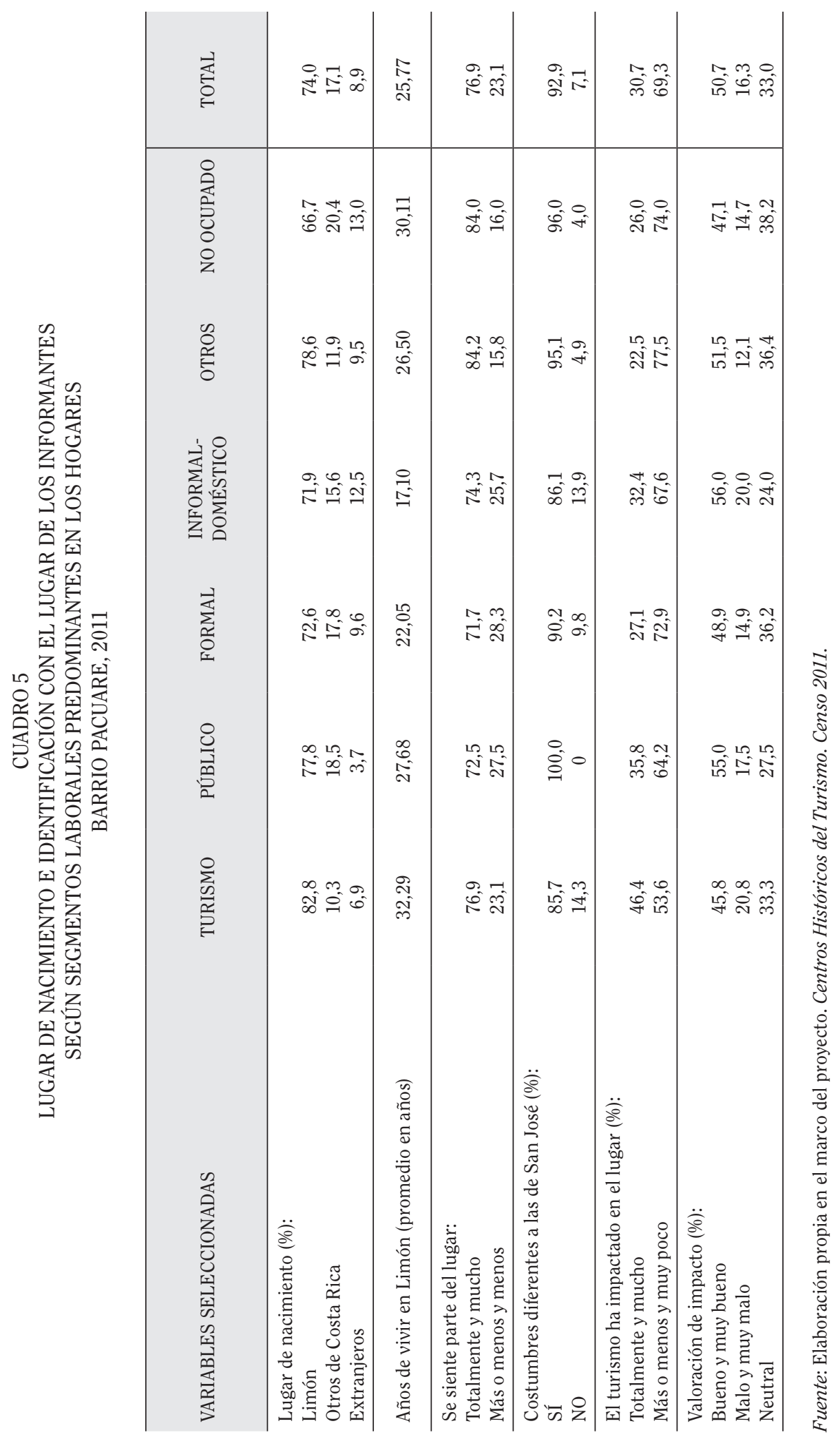


Antes de entrar propiamente en los datos sobre opiniones, en el cuadro 5, es importante resaltar con respecto a la procedencia de nacimiento de las personas que se desempeñan en los diferentes segmentos laborales, la cifra más alta la representa el "Turismo" (83\%); es decir, de todas las categorías es el "Turismo" la que alberga la mayor cantidad de personas porcentualmente hablando que son de la misma zona/ provincia. A nivel total, el $17 \%$ proceden de otras partes del país y el $9 \%$ son extranjeros. Además, es el "Turismo" la categoría que registra con más años promedio de vivir en Limón (32,2 años). Con respecto a las percepciones, se encontró que en todas las categorías, más del $70 \%$ se sienten parte del lugar bajo la categoría "Totalmente y mucho", siendo el "Turismo" la categoría con el dato más alto aunque no por mucho, esto sacando las categorías "Otros" y "No ocupados". En esta misma línea, se alberga una opinión de que las costumbres son diferentes a las de San José, donde se encontró un $100 \%$ de las personas en la categoría "Pública", $y$ en el caso del "Turismo", un $85 \%$. Mucho de esta respuesta puede estar asociada, tanto a la visión de comunidad, así como a la idea de separación político-discursiva y social que se señaló al inicio del documento, debido a los estereotipos $y$ dimensiones sociales para separar la región o integrarla limitadamente, donde se creó una lógica cultural diversa según las tradiciones y las condiciones socio-territoriales que existían.

Cuando se profundiza en la información de opiniones sobre el turismo y sus impactos, se observa que es precisamente la gente que trabaja en el sector "Turismo", la que señala con más peso: "Totalmente y mucho" $(46,4 \%)$. En las demás categorías no se supera el 36\%, se mantiene una idea de que "Más o menos y muy poco" ha sido el impacto del Turismo.

Esto podría explicarse por el nivel de contacto con la actividad que tienen unos frente a los otros; es decir, unos viviendo $y$ necesitando del turismo con más fuerza, mientras que en otros y otras, su dinámica no pasa necesariamente por la lógica turística. Sin embargo, sí se puede pensar en la significación que se le da al turismo desde todas las categorías, en el tema de impacto. Lo que faltaría precisar en otra oportunidad, es el tipo de impacto objetivo de la dinámica, cosa que ya se ha hecho en otros estudios y se podría cotejar para fortalecer la capacidad de comprensión del fenómeno. No obstante, si prosigue con la valoración subjetiva de las personas, se encuentra que en todas las categorías las personas señalan al turismo como "Bueno y muy bueno" y es precisamente el "Turismo", el que ahora se posiciona con el menor peso porcentual dentro de su categoría. En otras palabras, mientras que sobre "Bueno y muy bueno" el segmento: "Público" dio 55\%, "Formal" dio 49\% e "Informal-Doméstico" dio 56\%, "Otros" 51\% y "No ocupados" 47\%, el caso del "Turismo" dio 45,8\%, siendo el más bajo de todos y a su vez, es la tipología "Turismo" la que presenta el peso más alto en la respuesta "Malo y muy malo" (20,8\%) (esto se tiene que contemplar porque existe una tercera variante neutral que permite esta distribución). Esto se puede asociar que tal vez como se dijo, el estar vinculado al sector turístico, se pueden apreciar también las dificultades y las consecuencias con más profundidad y reconocer problemáticas que a vista general pasan desapercibidas, así como, las consecuencias sociales que no se entienden o vislumbran en el momento.

En el cuadro final (cuadro 6) se sigue ahondando en las valoraciones de las personas, rescatando ideas sobre costumbres, calidad de vida, construcción inmobiliaria, entre otros aspectos, con el sentido de clarificar algunos puntos sobre lo que opinan las personas de la Comunidad/Barrio Pacuare. Para la clasificación de las respuestas en todas las variables de dicho cuadro se tienen estas tres opciones: a) De acuerdo y más; b) En desacuerdo y más y c) Neutral, lo que permite, no sin cierta simplificación, conglomerar la información para ser presentada e interpretada. 


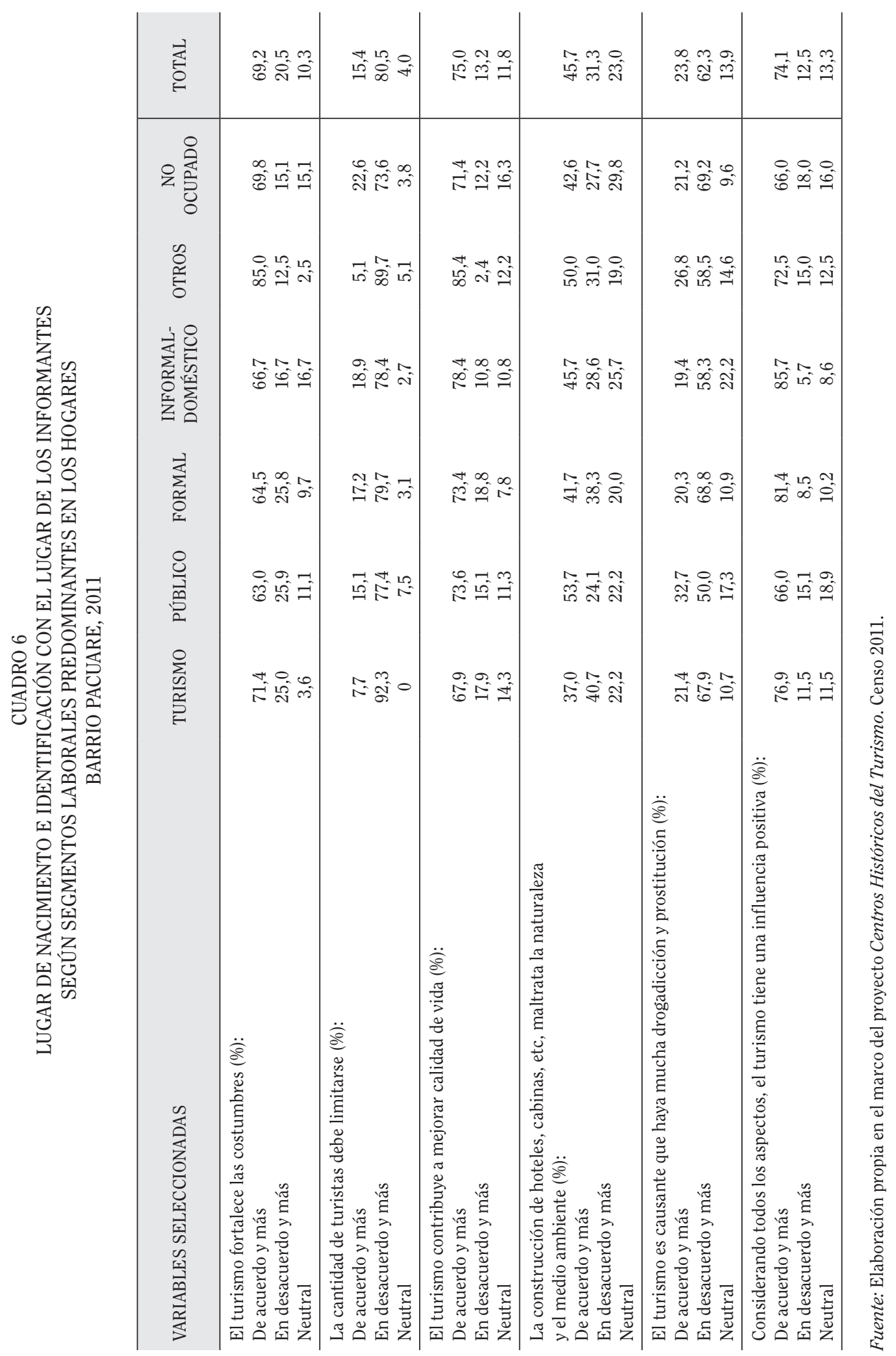


De toda esta gran cantidad de información procesada, interesa resaltar con puntualizaciones relacionales. La primera, a nivel general, es que las personas consultadas no quieren en su gran mayoría que se controle ni limite la cantidad de turistas (segunda variable). Esto podría deberse a una valoración inicial de impacto económico inmediato, pero no necesariamente conectado con el resto del proceso de utilización turístico. Esto habría que valorarlo con lo señalado anteriormente sobre el posible conocimiento (debido al vínculo que se tiene con el turismo), ya que resulta contradictorio el reconocimiento de lo "malo y muy malo" del impacto del turismo (20\% del cuadro 5), con el 92\% de "En desacuerdo y más" sobre el control y limitación de los turistas. Esto se podría deber a la valoración de la entrada de divisas por encima de otras circunstancias, como el daño ecológico, la alteración de la vida de la comunidad (del barrio), así como de la inversión extranjera que también tendría impactos socio-culturales y económicos en la misma vida de la comunidad. A esto se le pueden agregar los datos sobre el tema del desarrollo inmobiliario, donde solo el "Turismo" tiene datos superiores respecto a que la construcción de cabinas y hoteles no daña el medio ambiente (40,7\%, aunque no muy superior del $37 \%$ que sí señala el daño que se percibe). En las demás categorías sí hay una clara tendencia a señalar el daño ambiental con el desarrollo de este tipo de infraestructura.

En otro aspecto, las personas en los distintos segmentos laborales no asocian de manera alta el turismo con problemáticas como la drogadicción o la prostitución. Aunque, un concreto rubro aproximado de $20 \%$, sí encuentra una conexión con el turismo y en el caso del sector "Público", el número sube a un $33 \%$ que visualiza una relación entre esas dos problemáticas con el turismo. Esto se podría reflexionar desde la posición del funcionario público, que se despliega en una faceta laboral formal y donde las seguridades sociales están más presentes. Por otro lado, el turismo por vincularse en este caso a una faceta altamente informal, conecta esta incertidumbre con la generación de estas problemáticas como resul- tado de la dinámica de ausencia de trabajo y la entrada de nuevas formas y costumbres por medio del turismo.

En términos finales, las personas presentan un aspecto positivo general del turismo en la zona, en todas las categorías, con menores cambios en las desventajas. Esto se revela en la última variable del cuadro 6 , considerando las demás variables del cuadro y del resto de variables del instrumento que no necesariamente se muestran en estos datos. Sobresale que es el sector "Informal-Doméstico" (86\%) y "Formal" $(81,4 \%)$ los que tienen los datos más positivos sobre la influencia positiva, $y$ en tercer lugar vienen los trabajadores que se desempeñan en la misma rama turística (77 \%). Lo que deja ver una panorámica no solamente positiva, sino de oportunidad, de complementariedad en varios niveles de la tipología desarrollada y que muestra una faceta económica que se vislumbra como prometedora, pese a las valoraciones que muestran recelos sobre la dinámica.

\section{CONCLUSIONES}

En el artículo se realizó la presentación de una serie de datos acerca de una tipología de segmentos laborales, donde una de las justificaciones es estudiar el turismo dentro de una dinámica laboral en el Barrio Pacuare de Limón. Dentro de las consideraciones generales está el rescatar la opinión extendida en los distintos segmentos estudiados, respecto a apoyar y ver con buena lid la incorporación, inclusive sin restricciones de la cantidad de turistas, dentro de la zona socio-geográfica de Pacuare. Además, se aprecia una diferenciación en términos de las jefaturas en el caso del segmento "Turismo" y en el "Informal-Doméstico" (este último esperable por la condición muchas veces de madres solteras), donde las mujeres son las que primordialmente llevan las jefaturas en los hogares, diferente al resto de segmentos laborales.

La idea de abordar la comunidad redunda en tratar de acoplar explicativamente vínculos particulares (familiares $y$ de compadrazgo, entre otros), pero que se encuentra en diálogo $y$ conflicto con pautas productivas globalizadas, como es el caso del tipo de turismo que se ha desarrollado en Costa Rica, donde muy 
sucinta e inicialmente se puede observar una percepción (sin entrar en una discusión teórica sobre el concepto de percepción) positiva $y$ de apoyo al turismo, sin necesariamente estar del todo al tanto de situaciones conflictivas y las consecuencias. Además, valorando las posibles contradicciones entre el pensar, la necesidad y el hacer, que pueden estar afrontando personas de la zona, ya que pese a poder detectar contradicciones entre lo que hacen y el medio ambiente $y$ el cultural (con niveles negativos para esos rubros), se ven en la necesidad de continuar con dicha lógica productiva y que a su vez, en la opinión expresada en el Censo y a nivel público, también expresan el apoyo. Por esta razón, es importante mantener un matiz de prudencia crítica respecto a los datos y lo que sugieren, así como, lo que potencian en el análisis respecto a las subjetividades.

El turismo es un área que mantiene una posición significativa en pesos y que se posiciona como una entrada económica importante para un grupo de personas y que sin la posibilidad de dar una respuesta certera, se avizoran posibles contactos y conexiones que no necesariamente son visibles por medio del censo, por lo que un trabajo cualitativo complementaría este estudio con una visión de contraste $y$ aporte en la calidad explicativa del fenómeno del turismo en la zona.

\section{FUENTES DE INFORMACIÓN}

Bonilla Soto, P.; Pérez Díaz, J. y Michelena Velásquez, E. Aprovechamiento del recurso hídrico como fuente ecoturística "Aguas del Zurquí". Limón, Costa Rica: Universidad Sede Regional Limón Dr. Rómulo Salas Guevara de la Universidad de Costa Rica, 2005.

Bialakowsky, Alejandro. "Comunidad y sentido en la teoría sociológica contemporánea: las propuestas de A. Giddens y J. Habermas". Papeles del ceIc 53. Universidad de Buenos Aires, 2010. En: <http://www.identidadcolectiva.es/ pdf/53.pdf $>$ [consultado el 27 de agosto de 2012].

Cordero, Allen. "Los viejos y olvidados centros históricos del turismo: algunas evidencias para el caso costarricense". ¿Es posible otro turismo? Su realidad centroamericana, nueve casos de estudio. Allen Cordero y Paul Bodson (eds.). San José, Costa Rica: flacso Costa Rica-Colección Pensamiento Social, 2011.

Cordero, Allen. Nuevos ejes de acumulación y naturaleza: El caso del turismo. San José, Costa Rica: flacso, 2004.

García, P. y Lepe, I. "Sostenibilidad turística. Un análisis del marco jurídico turístico costarricense $y$ del escenario turístico de la provincia de Limón/Costa Rica". Revista Intersedes X. Universidad de Costa Rica, 2009: 39-61.

Murillo, Ericka. "Desarrollo turístico "Agu trot lodge" en el cerro de Tortuguero". [Trabajo final de Graduación sometido a la consideración de la comisión del Programa de Estudios de Posgrado en Arquitectura para optar al grado de Magíster]. San José, Costa Rica: Universidad de Costa Rica, 2006.

Pérez Sáinz, J.P.; Andrade-Eekhoff, K.; Bastos, S. y Herradora, M. La estructura social ante la globalización. Procesos de reordenamiento social en Centroamérica durante la década de los 90. San José, Costa Rica: FLACSO-ONu-CEPAL, 2004.

Poviña, Alfredo. "La idea sociológica de 'comunidad"'. Actas del Primer Congreso Nacional de Filosofía. Mendoza, Argentina. Universidad de Buenos Aires, 1949: 1757-1763. En: <http://www. filosofia.org/aut/003/m49a1757.pdf> [consultado el 27 de agosto de 2012].

Pilquiman, Maricela y Skewe, Juan Carlos. "Relatos en torno al etnoturismo: la comunidad indígena de ChoroyTraigén, Provincia de Osorno, y sus proyectos de desarrollo". Revista Líder 16 (12). 2010: 105-124. En: $<$ http://ceder. ulagos.cl/lider/images/ 
numeros/16/\%5BLIDERVol16A\%C3\%B1o122010 - Is s - 0717 - $0165 \% 5$ D 7 . Relatosentornoaletnoturismo.pdf $>$ [consultado el 26 de agosto de 2012].

Román, L.; Jiménez, L. y Molina, P. "Análisis $y$ evaluación de las fuentes de financiamiento y crédito para la microempresa turística: estudio de caso de Cahuita y cantón central de Limón." [Tesina de Grado para optar por el grado de bachillerato en Turismo Ecológico]. Limón, Costa Rica: Universidad Sede Regional Limón Dr. Rómulo Salas Guevara de la Universidad de Costa Rica, 2004.

Ruiz, Adolfo M. "Zona de cooperación y régimen de turismo: el caso de la zona de turismo sustentable del Caribe (zTsc), 1989-2005". [Tesis sometida a la consideración de la
Comisión del Programa de Doctorado en Gobierno y Políticas Públicas para optar por el grado de Doctor en Gobierno y Políticas Públicas]. Universidad de Costa Rica. Ciudad Universidad Rodrigo Facio, 2006.

Vanegas, Mercedes. "Ecoturismo instrumento de desarrollo sostenible". [Monografía para optar por el título de Especialista en Gestión Ambiental]. Medellín, Colombia. Universidad de Antioquia, 2006. En: $<$ http:/tesis.udea.edu.co/dspace /bitstream/10495/149/1/EcoturismoInstrumentoDesarrolloSostenible.pdf> [consultado el 26 de agosto de 2012].

Fecha de ingreso: 01/11/2012 Fecha de aprobación: 10/05/2013 
